\title{
CFD: Computational Fluid Dynamics or Confounding Factor Dissemination? The Role of Hemodynamics in Intracranial Aneurysm Rupture Risk Assessment
}

J. Xiang, V.M. Tutino, K.V. Snyder, and H. Meng

o- $=$

\begin{abstract}
SUMMARY: Image-based computational fluid dynamics holds a prominent position in the evaluation of intracranial aneurysms, especially as a promising tool to stratify rupture risk. Current computational fluid dynamics findings correlating both high and low wall shear stress with intracranial aneurysm growth and rupture puzzle researchers and clinicians alike. These conflicting findings may stem from inconsistent parameter definitions, small datasets, and intrinsic complexities in intracranial aneurysm growth and rupture. In Part 1 of this 2-part review, we proposed a unifying hypothesis: both high and low wall shear stress drive intracranial aneurysm growth and rupture through mural cell-mediated and inflammatory cell-mediated destructive remodeling pathways, respectively. In the present report, Part 2, we delineate different wall shear stress parameter definitions and survey recent computational fluid dynamics studies, in light of this mechanistic heterogeneity. In the future, we expect that larger datasets, better analyses, and increased understanding of hemodynamicbiologic mechanisms will lead to more accurate predictive models for intracranial aneurysm risk assessment from computational fluid dynamics.
\end{abstract}

ABBREVIATIONS: CFD = computational fluid dynamics; IA = intracranial aneurysm; LSA = low shear-stress area; $M$ WSS = maximum wall shear stress; OSI = oscillatory shear index; TAWSS = time-averaged wall shear stress; WSS = wall shear stress

I mage-based computational fluid dynamics (CFD) holds a prominent position in the patient-specific evaluation of intracranial aneurysms (IAs). Hemodynamic parameters, particularly those related to aneurysmal wall shear stress (WSS), the frictional force of flowing blood on the vessel lumen, have been shown to correlate with IA growth ${ }^{1-3}$ and rupture. ${ }^{4,5}$ Consequently, the clinical community has taken an interest in not only the intuitive and esthetic aspects of CFD simulations (dubbed "color for doctors") ${ }^{6}$ but also the enormous potential of CFD to provide objective, quantitative, and mechanism-based markers of aneurysm rupture risk.

In a recent American Journal of Neuroradiology editorial, Kallmes $^{7}$ raised concerns regarding the current status of CFD studies and their clinical utility in IA rupture risk assessments,

From the Toshiba Stroke and Vascular Research Center (J.X., V.M.T., K.V.S., H.M.) and Departments of Neurosurgery (J.X.), Biomedical Engineering (V.M.T.), and Mechanical and Aerospace Engineering (H.M.), University at Buffalo, State University of New York, Buffalo, New York.

Grant support (H.M.) was provided by the National Institutes of Health (5R01NS064592) and Toshiba Medical Systems.

Please address correspondence to Hui Meng, PhD, Toshiba Stroke and Vascular Research Center, University at Buffalo, Clinical and Translational Research Center, 875 Ellicott St, Buffalo, NY 14203; e-mail: huimeng@buffalo.edu

- Indicates open access to non-subscribers at www.ajnr.org

三 Indicates article with supplemental on-line tables

http://dx.doi.org/10.3174/ajnr.A3710 asking if CFD should alternatively be interpreted as "confounding factor dissemination.” This thought- and debate-provoking question reflects wide concerns over the growing number of hemodynamic parameters and conflicting findings reported in the current literature. Indeed, 3 other editorials published in the same jour$\mathrm{nal}^{8-10}$ appeared shortly after Kallmes's editorial. Cebral and Meng ${ }^{8}$ offered the first counterpoint, in which they commented that inconsistencies in hemodynamic parameter definitions and conflicting findings reflect exploratory efforts of isolated groups with small datasets; yet, more importantly, the conflicting findings could originate from the intrinsic complexity of aneurysm growth and rupture mechanisms and our scarce knowledge about them. Intracranial aneurysm has long been suspected to be a highly heterogeneous disease, containing several disease types. ${ }^{10,11}$ As Strother and Jiang ${ }^{10}$ pointed out in their editorial, IA may even be a spectrum of a disease or even multiple separate diseases.

Currently, conflicting results concerning aneurysmal WSS, the most frequently explored hemodynamic parameter of augmented rupture risk, puzzle the IA research and clinical communities. Both high and low WSS have been found to be correlated with IA growth and rupture. ${ }^{1-5,12,13}$ Prompted by this "high-versus-low WSS" controversy, we published Part 1 of a 2-part review, in which we proposed a unified hypothesis regarding the roles of high and low WSS in IA growth and rupture. ${ }^{11}$ That review aimed 
at providing new insight into the complex hemodynamic-biologic mechanisms underlying IA pathophysiology. In brief, we proposed that both high and low WSS could drive IA growth and rupture. On the basis of published studies on pathologic vascular responses to hemodynamics in IA genesis ${ }^{14,15}$ and atherosclerosis, ${ }^{16}$ we hypothesized that these 2 aberrant hemodynamic conditions could elicit different destructive vascular remodeling pathways, mediated by mural cells and inflammatory cell infiltrates, respectively. Such diversity in the driving hemodynamics and elicited biologic pathways may generate the observed heterogeneity of IA phenotypes. Understanding this intrinsic mechanistic complexity could help us to better characterize and converge inconsistent CFD findings.

To follow up, this work serves as Part 2 of this 2-part review. We delineate commonly used definitions of WSS and related parameters, provide a detailed survey on how WSS can be defined and used in image-based CFD studies, and describe how it has been related to IA growth and rupture. In conjunction with Part 1 , we hope this review sheds light on the disparate CFD findings concerning the role of WSS in IA growth and rupture.

\section{Current Status of Image-Based CFD Studies of Aneurysmal Hemodynamics}

Hemodynamics plays a central role throughout IA natural history. ${ }^{11}$ Because of a lack of appropriate animal models to study hemodynamic-biologic mechanisms, investigators have resorted to the use of CFD analysis of patient IA images ${ }^{1-5,12,13,17-38}$ to identify hemodynamic characteristics and explore potential mechanisms associated with IA growth and rupture. The capability of image-based CFD to investigate detailed 3D aneurysmal hemodynamics, combined with the availability of segmentation tools such as the Vascular Modeling Toolkit (www.vmtk.org), ${ }^{39}$ has contributed to an increasing number of hemodynamic IA studies over the past 2 decades. There have been several large, multi-nation projects that use image-based CFD to study aneurysms. For example, the @neurIST project, with a €17 million budget, gathered data from 28 public and private institutions in 12 European countries from $2006-2010 .^{40-42}$

Over the past 10 years, the number of publications in this field has increased nearly exponentially, ${ }^{9}$ as image-based CFD studies have grown broader and deeper. Simulations have steadily migrated from idealized geometries to patient-specific IA geometries, from steady-state flow to pulsatile flow, and from qualitative flow pattern descriptions 20,22,23,43 $^{2}$ sophisticated quantitative statistical analyses. ${ }^{4,5,30}$ Furthermore, efforts to bolster statistical power have increased the number of patient-specific IA cases per hemodynamic study from 1 (in the year 2003) ) $^{44}$ to 210 (in the year 2011). ${ }^{5}$ Worldwide interactions between engineers and clinicians, exemplified by the annual International Intracranial Stent Meeting (http://www.ics-meeting.net), have stressed the dire medical need for clinically relevant information, including rupture risk assessments.

The rising number of CFD studies has resulted in a growing number of proposed hemodynamic parameters related to IA growth and rupture. On-line Table 1 summarizes the most commonly defined hemodynamic parameters in these studies. Clearly, WSS has been the focus because it is a measurable biome- chanical factor (obtained from CFD calculation) that holds the promise of reporting and predicting IA development.

\section{WSS in Aneurysm Development}

WSS and Pressure in IA Pathologic Remodeling. Wall shear stress directly acts on the luminal surface of vascular endothelial cells to stimulate biologic responses through the main stages of IA natural history. As shown in Fig $2 A$ of Part $1,{ }^{11}$ blood flow interacts with the IA wall by exerting both pressure and WSS. Blood pressure elicits tensile stresses in the wall, which, when unbalanced, stimulate medial cell-mediated collagen synthesis and cross-linking and collagen degradation. ${ }^{45}$ Wall shear stress, on the other hand, is sensed by endothelial cells, which transduce this mechanical signal into biologic signals, activating biochemical pathways that maintain vascular homeostasis and regulate remodeling. ${ }^{46}$ Aberrant levels of WSS elicit endothelial cell-mediated pathologic responses, including pro-inflammatory behavior, ${ }^{16}$ matrix metalloproteinase activation, ${ }^{47}$ cell death, ${ }^{48}$ extracellular matrix degradation, and destructive remodeling. ${ }^{15,49,50}$

Although both tensile stress and WSS can affect aneurysmal pathobiology, the current exploration of WSS-mediated effects appears to offer a greater clinical utility for the following reasons: 1) Patient-specific in vivo flow dynamic simulation, that is, CFD, and WSS analyses can be routinely performed from existing medical imaging modalities (DSA, CTA, MRA). Conversely, tensile stress calculation requires knowledge of patient-specific, in vivo wall thickness and material properties, which are unavailable from current imaging capabilities. 2) The simplified analyses of tensile stress-mediated pathways involve collagen turnover in response to imbalanced wall stresses. Theoretically, this process should reduce the imbalance and ultimately lead to IA stabilization, not to unbound growth or rupture. ${ }^{51}$ The rupture event itself, a catastrophe that results when wall stress exceeds wall strength, is precipitated by a local weakening of the IA wall resulting from pathologic remodeling. ${ }^{45}$ Without the means to monitor wall stress and wall strength, it is impossible to predict when an IA will rupture. However, because pathologic remodeling is partially mediated by abnormal WSS, which can persist throughout IA natural history, WSS may be a suitable for clinical application at this stage.

Various Definitions of WSS. By definition, WSS is a mechanical stress that acts tangential to the vessel lumen. For unsteady flow (which is always the case for patient aneurysms), instantaneous WSS can change its magnitude and direction with space and time. Motivated by different hypotheses regarding the hemodynamic conditions driving IA progression, CFD researchers have explored different variations of WSS definitions.

To reflect the hypothesis that lasting exposure to abnormal WSS potentiates wall weakening, WSS has been most commonly reported as the time-averaged magnitude of WSS (the mathematical definition is given in the first row of On-line Table 1), or TAWSS. Time-averaged wall shear stress is defined point-wise on the lumen of the vessel and plotted as a surface distribution. Unless explicitly stated otherwise, time-averaging over 1 cardiac cycle is usually implied. Occasionally, however, instantaneous WSS at a 
certain time point in the cardiac cycle, such as peak systole or diastole, is used instead of TAWSS.

To compare the overall level of WSS among different aneurysms for risk stratification, TAWSS is sometimes further spatially averaged over the entire aneurysm sac, such that each aneurysm has one average aneurysmal WSS value. ${ }^{5,30,31}$ Alternatively, some authors prefer to examine the spatial maximum (MWSS in Online Table 1) of TAWSS in an aneurysm. Both values require identification of the IA neck and an objective method to isolate the aneurysm. ${ }^{52,53}$

To remove the dependence of WSS values on the inlet flow condition, the time- and space-averaged aneurysmal WSS can be normalized by the average parent vessel WSS value to become a nondimensional number ${ }^{4}$ (second row in On-line Table 1). It has been suggested that normalized WSS is more reliable than the absolute WSS when used to stratify IA rupture risk, ${ }^{4}$ especially when patient-specific inlet flow conditions are unavailable and CFD simulations are carried out under assumed inlet flow conditions. When surrogate (assumed) inlet flow boundary conditions are used, the CFD-generated absolute aneurysmal WSS values are not meaningful; only relative values are meaningful. Normalization by parent vessel WSS generated from the same CFD simulation minimizes the dependence on inlet conditions. Furthermore, this normalized ratio compares the pathologic IA wall with the healthy parent vessel wall, as a baseline. In parallel to such approaches in hemodynamic studies, recent morphologic studies have shown that to better inform rupture risk, aneurysm size should be evaluated relative to the parent vessel diameter (thus generating an important new morphologic metric, size ratio). ${ }^{54}$ Size ratio is potentially better at predicting IA rupture risk than aneurysm size only. ${ }^{4,54-56}$ Similarly, aneurysmal WSS values might be better evaluated against a healthy baseline from the individual at a specific location, for example, WSS values from the parent vessel.

Besides responding to TAWSS directly, endothelial cells respond to temporal oscillations and spatial gradients of WSS. ${ }^{16,57,58}$ The hypothesis that directional variations of WSS induce pathologic endothelial cell responses (as in atherogenesis) is reflected by the oscillatory shear index (OSI), a dimensionless metric of directional changes in WSS ${ }^{59}$ (third row in On-line Table 1). Oscillatory shear index is often used to describe the disturbance of a flow field. Additionally, spatial WSS gradient, the derivative of WSS along the flow direction, is also considered in some studies. ${ }^{4}$ Variations of these factors, for example, low shearstress area (LSA) ${ }^{4}$ and gradient oscillatory number factor (GON factor), ${ }^{60}$ are summarized in On-line Table 1.

It is important to recognize that instantaneous WSS is a vector. During a cardiac cycle, the unsteady aneurysmal flow leaves imprints of flickering, swirling, and dancing WSS vectors on the luminal surface, forming various patterns such as sources, sinks, or streets. The definitions of WSS-derived hemodynamic factors above are degenerated forms that account only for the magnitude of WSS (as in TAWSS) or the magnitude of the cycle-averaged vector (as in OSI). How endothelial cells respond to various patterns of instantaneous WSS vectors is unknown but could play an important role in the pathologic remodeling leading to IA growth and rupture. When new evidence indicates such roles, new hemo- dynamic parameters capturing these characteristics may need to be defined.

Currently, a plethora of WSS definitions and other flow parameters are confusing to both the clinical and engineering communities. ${ }^{7-10}$ Findings from different studies correlating WSS to IA progression are not converging: both high and low WSS are correlated to growth and rupture. To gain a better understanding of these divergent findings, we systematically review the current literature on hemodynamic studies correlating high and low WSS to IA growth and rupture in the following 2 sections.

\section{CFD Studies of High and Low WSS in Aneurysm Growth}

The investigation of IA growth requires longitudinally following patients with 3D imaging. Because of a lack of reliable risk assessment systems, discovered IAs are often defensively treated, leaving only a small number of aneurysms for conservative observation and periodic imaging. ${ }^{1,2,17}$ These IAs tend to be small or have no clear, safe surgical options. ${ }^{1,2,17}$ Thus, only a limited number of studies have been able to investigate the hemodynamics of growing IAs.

In On-line Table 2, we summarize 5 published studies that investigated patient-specific hemodynamics during IA growth. ${ }^{1-3,17,19}$ The number of patients in these studies ranged from 1 to 25 . Impressively, $4^{1-3,17}$ of the 5 studies used patient-specific inlet boundary conditions (velocity from phase-contrast MRA), which is more accurate for CFD simulation than the widely used, assumed inlet flow conditions.

Three of these studies found a correlation between IA growth and low aneurysmal WSS. Jou et $\mathrm{al}^{1}$ demonstrated this correlation in their CFD analysis of 2 aneurysms. Additionally, a serial study containing baseline imaging and 4 follow-ups by Acevedo-Bolton et $\mathrm{al}^{2}$ found that the region of maximal growth in a giant basilar trunk IA consistently had the lowest WSS (Fig 1A). ${ }^{2}$ The same group analyzed 7 growing aneurysms in a follow-up study and found a linear correlation between the local displacement of the IA wall (that is, enlargement) and low WSS, as shown in Fig $1 B .{ }^{17}$

Studies by Sugiyama et $\mathrm{al}^{3}$ and Sforza et al, ${ }^{19}$ however, found that both low WSS and high WSS were associated with aneurysm growth. Sugiyama et $\mathrm{al}^{3}$ reported 2 adjacent growing aneurysms in 1 patient, each with different hemodynamic characteristics and growing patterns. They found that the distal growing aneurysm was associated with low and oscillatory WSS in the entire aneurysm sac, whereas the proximal aneurysm was subjected to local high flow in the growing lobe. ${ }^{3}$ In the other study, Sforza et $\mathrm{al}^{19}$ followed several growing aneurysms. The growth of 3 aneurysms was correlated with high WSS impingement at the dome, whereas the growth of 2 aneurysms was correlated with low WSS at the dome.

These inconsistent findings may be attributed to a limited number of cases and thus selection biases. However, we believe that these contradictions may stem from the intrinsic mechanistic complexity of IAs. As hypothesized in Part $1,{ }^{11}$ both high and low WSS could drive IA progression. To confirm this, future investigations of IA growth would need to collect a larger volume of longitudinal data despite the inherent difficulties in building large, unskewed databases of unruptured growing IAs. One possible solution would be to implement population-based screening 

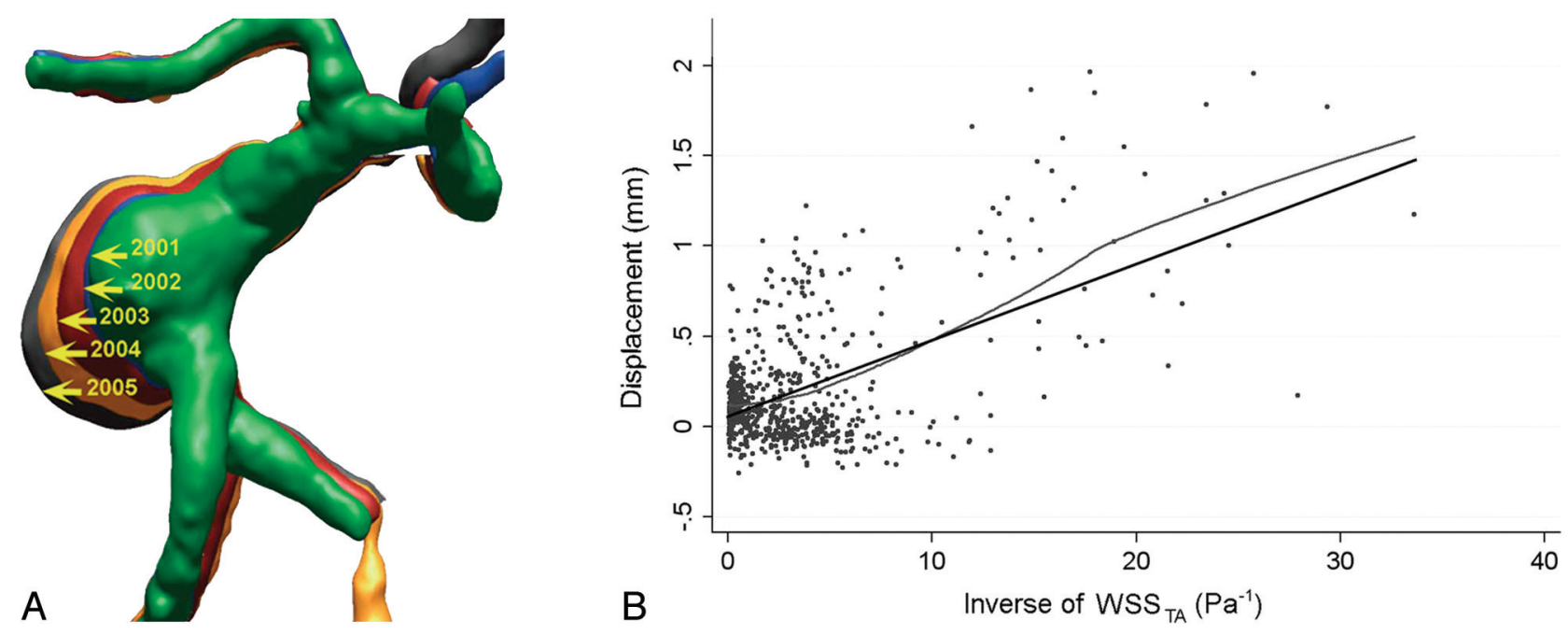

FIG 1. Longitudinally followed aneurysms show correlation between growth and low WSS. A, Overlying 3D images of a basilar-trunk aneurysm from consecutive years show dramatic size increase in the direction of the lowest WSS. ${ }^{2}$ B, Linear correlation between local displacement of IA wall (IA enlargement) and inverse of time-averaged WSS $(P<.001)$ in a follow-up study analyzing 7 growing IAs by the same group. ${ }^{17}$ Reproduced with permission from Acevedo-Bolton G, Jou LD, Dispensa BP, et al. Estimating the hemodynamic impact of interventional treatments of aneurysms: numerical simulation with experimental validation: technical case report. Neurosurgery 2006;59:E429-30 and Boussel L, Rayz V, McCulloch C, et al. Aneurysm growth occurs at region of low wall shear stress: patient-specific correlation of hemodynamics and growth in a longitudinal study. Stroke 2008;39:2997-3002.

methods. For example, in Japan, screening of the population to detect and monitor IAs has provided the opportunity to longitudinally follow unruptured aneurysms. However, these conservatively followed IAs tend to be small, because large and giant IAs are probably treated rather than monitored. ${ }^{28,31}$

\section{CFD Studies of High and Low WSS in Aneurysm Rupture}

Whereas longitudinal data are needed to study aneurysm growth, cross-sectional data have been used to study IA rupture, accounting for a larger number of rupture risk assessment studies. By extracting hemodynamic features of ruptured IAs and comparing them with those of unruptured IAs, these studies implicitly assume that unruptured aneurysms resembling ruptured aneurysms are at higher risk of rupture than those that do not. Thus, hemodynamic parameters that significantly distinguish ruptured from unruptured IAs have been explored and suggested as potential markers for rupture risk.

In On-line Table 3, we chronologically summarize 24 studies that performed CFD on patient-specific aneurysm geometries in search of correlations between aneurysmal hemodynamics and rupture. The number of aneurysm cases in each study ranged from 6-210. Whereas all 24 studies included pulsatile CFD simulations, none used patient-specific flow waveforms at inlet boundaries. As for the specification of inlet flow magnitude, different authors adopted different assumptions across different cases, namely the same flow velocity, ${ }^{12,27,33,34}$ the same flow rate, ${ }^{4,13,20,28,30,31,35}$ or the same WSS., ${ }^{52-26,43,61}$ The specified values were either taken from the literature or measured from one subject. Because the inlet boundary condition specified in this way is not patient-specific, absolute levels of physical quantities, such as WSS, calculated from CFD are not accurate. However, the distributions and relative levels of these hemodynamic quantities and flow patterns should not be significantly influenced by the inlet condition. ${ }^{5,13}$
Although many hemodynamic parameters have been proposed and analyzed, most of these studies have focused on WSS. Published results regarding whether aneurysmal WSS can significantly distinguish ruptured from unruptured aneurysms are conflicting. These findings typically fall into 4 categories: 1) high spatial mean WSS is correlated with rupture ${ }^{5}$;2) low spatial mean WSS is correlated with rupture $e^{4,27,29}$; 3) high maximum WSS is correlated with rupture ${ }^{22,23}$; and 4) WSS is not correlated with rupture. ${ }^{13,28,30}$ Examples of low WSS correlated with aneurysm rupture are presented in Fig $2 .{ }^{4}$ Caution is required when interpreting results from these studies because many were based on small datasets and consequently have inherent selection bias.

Below, we discuss 2 notable studies that investigated a large number of IAs but produced inconsistent findings. ${ }^{4,5}$ Xiang et $\mathrm{al}^{4}$ published the first study that comprehensively examined multiple IA hemodynamic factors (as well as morphologic factors) in a large dataset. The authors analyzed 119 aneurysms and found that among the 7 hemodynamic parameters examined, 6 were significantly correlated with ruptured IAs. These were lower WSS, lower maximum WSS (MWSS), higher relative residence time, higher OSI, higher LSA, and larger number of vortices. Only 1 parameter, WSS gradient, showed no significance. Further multivariate logistic regression reduced the list of significant parameters to 2 independently significant parameters: WSS and OSI. The odds of an IA being ruptured can be estimated by use of a prediction model, which is an exponential function with (-WSS) and OSI in the exponent. According to this result, aneurysms with lower WSS and higher OSI have a larger chance of being ruptured. ${ }^{4}$

The essential finding that low WSS and high OSI are correlated with IA rupture is consistent with recent clinical results by Omodaka et al. ${ }^{35}$ By intraoperatively identifying rupture points of 


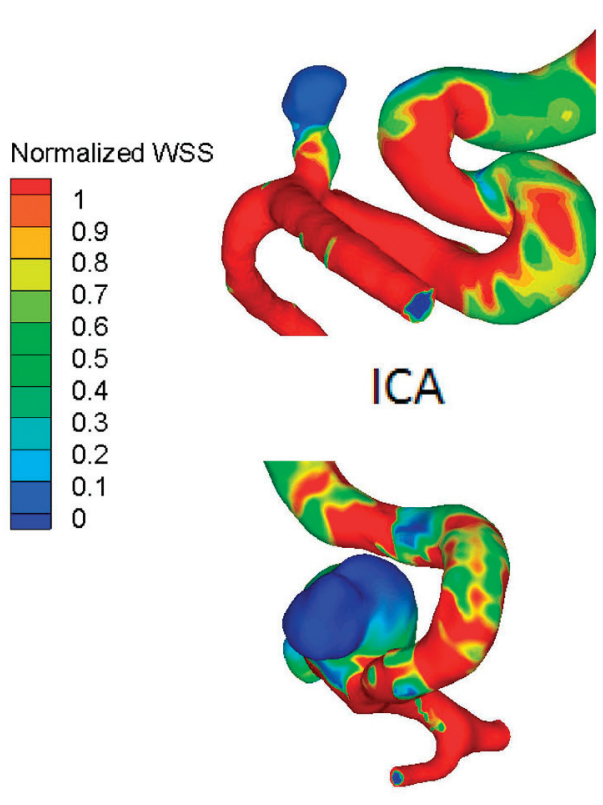

ICA

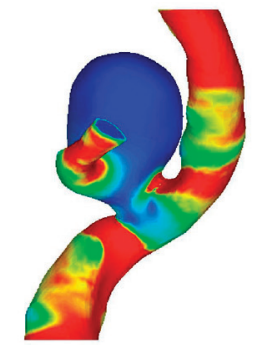

PCA

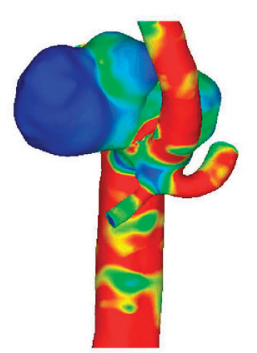

MCA

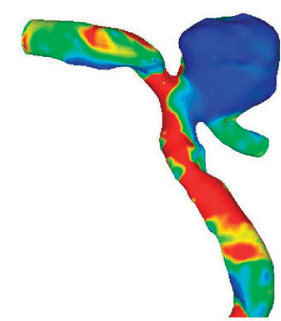

MCA

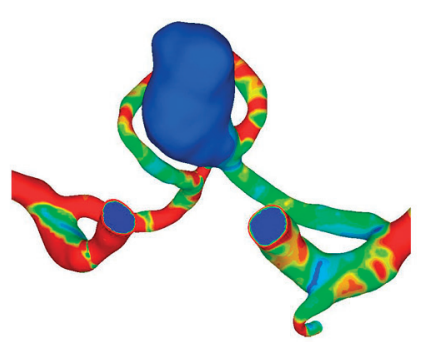

ACOM

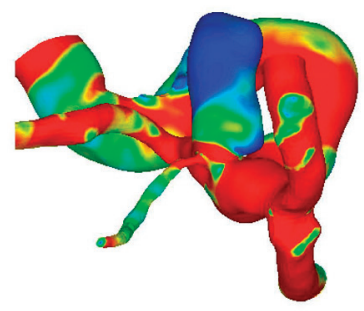

PCOM

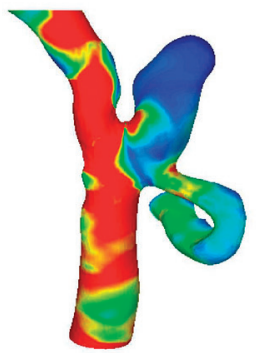

ACA

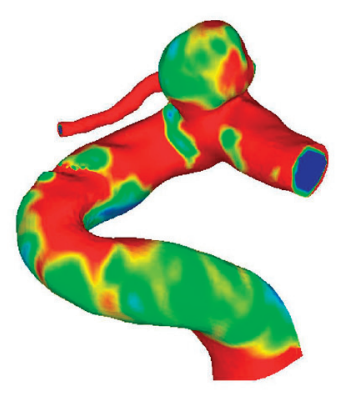

ICA

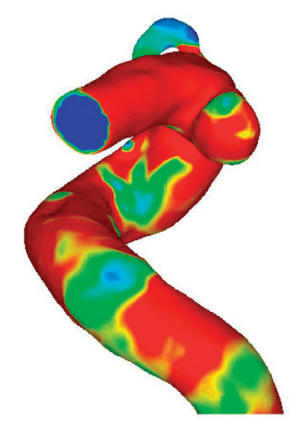

ICA

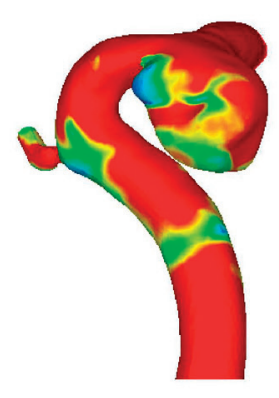

ICA

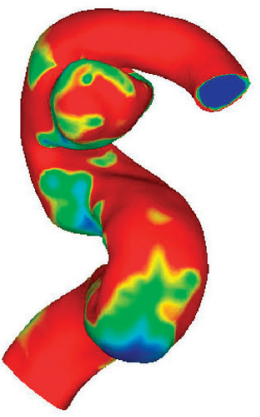

ICA

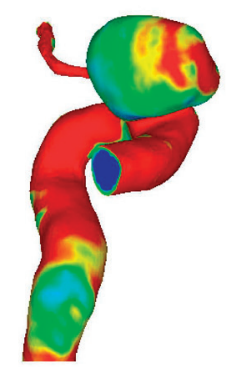

ICA-Oph

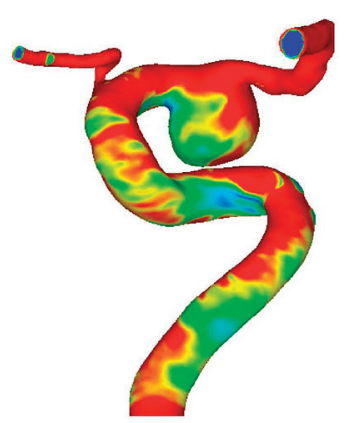

ICA-Oph

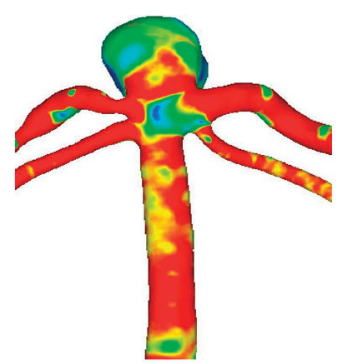

BA

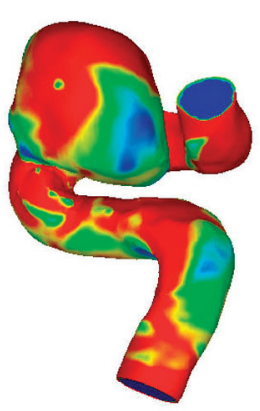

ICA

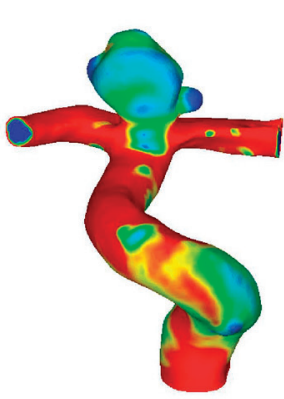

ICA

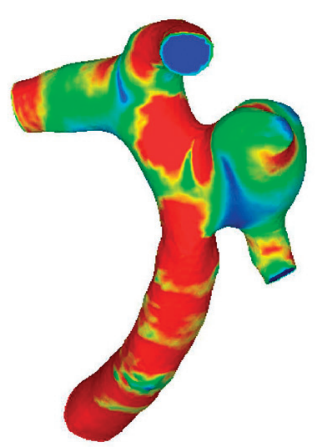

MCA

FIG 2. Examples of WSS distributions in ruptured (top 2 rows) and unruptured aneurysms (bottom 2 rows) from a study of 119 aneurysms by Xiang et al. ${ }^{4}$ Ruptured aneurysms were associated with lower aneurysmal WSS $(P<.0001)$. Reproduction with permission from Xiang J, Natarajan SK, Tremmel M, et al. Hemodynamic-morphologic discriminants for intracranial aneurysm rupture. Stroke 2011;42:144-52

6 MCA IAs and then performing retrospective CFD analysis, they found that rupture points were located in regions of lower WSS and higher OSI, compared with surrounding areas. Interestingly, they found that by numerically removing blebs within the IA, areas of bleb formation were associated with flow impaction and were subjected to higher WSS than surrounding areas. Although the number of cases in this study was relatively small, their findings support the hypothesis that low WSS could be causing the wall to weaken at rupture points.

Another large study, however, arrived at different conclusions. Cebral et $\mathrm{al}^{5}$ analyzed 210 aneurysms and investigated 7 hemodynamic parameters. From univariate statistical analysis, they found 
that 4 of the 7 parameters, that is, larger MWSS, larger inflow concentration index and larger shear concentration index, and lower viscous dissipation ratio, were significantly correlated with ruptured IAs. The other 3 hemodynamic parameters, that is, LSA, kinetic energy ratio, and low shear index, did not achieve statistical significance. By simulating these 210 aneurysms with different inlet flow conditions ( 2 different pulsatile conditions and 3 steady-flow conditions), the authors further demonstrated that though the values of hemodynamic quantities changed with different inlet flow conditions, the statistical differences or ratios between their mean values over the ruptured and unruptured IAs were maintained.

In the above-mentioned large studies (Xiang et $\mathrm{al}^{4}$ and Cebral et $\mathrm{al}^{5}$ ), findings regarding LSA and MWSS, the only overlapping parameters analyzed, were contradictory. Low shear stress area significantly separated ruptured from unruptured IAs in the Xiang et $\mathrm{al}^{4}$ study but not in the Cebral et $\mathrm{al}^{5}$ study. This difference could partially be attributed to the different definitions of LSA used by these authors. Xiang et $\mathrm{al}^{4}$ used $10 \%$ of parent vessel WSS value as the threshold for low WSS in LSA calculation, whereas Cebral et $\mathrm{al}^{5}$ used a threshold below 1 standard deviation of parent vessel WSS. The conflicting results could also reflect different hemodynamic mechanisms dominating these 2 IA cohorts. Overall, their inconsistent LSA definitions make it difficult to compare their results.

In the case of MWSS, which had consistent definitions in both studies, the reported opposite associations of MWSS with rupture might suggest something more fundamental. Xiang et $\mathrm{al}^{4}$ found that low MWSS along with low (average) WSS were significantly correlated with ruptured IAs. Furthermore, Xiang et $\mathrm{al}^{4}$ found that in most of their IAs, the MWSS was located near the aneurysm neck. However, Cebral et $\mathrm{al}^{5,20,22,23}$ found that high MWSS correlated with ruptured IAs and was mostly located in the IA dome. This raises an important question: did these 2 cohorts contain vastly different types of aneurysms, which might have been subjected to different flow and rupture mechanisms?

In a recent editorial, Strother and Jiang ${ }^{10}$ suggested that IA may not be one single disease but may be a spectrum of a disease or even multiple diseases. In Part 1 of our 2-part review, ${ }^{11}$ we hypothesized that there are 3 principal IA phenotypes: Type I, typically small IAs with thin, translucent walls, dominated by high WSS and impinging flow; Type II, typically large IAs with thick and atherosclerotic walls, dominated by low WSS and disturbed flow; and a third type, a combination of Type I and Type II, containing both high and low WSS. We proposed that both high and low WSS can drive IA growth and rupture by different biologic mechanisms: high WSS may trigger a mural cell-mediated destructive remodeling pathway, akin to the process of IA gene$\operatorname{sis}^{14,15}$ or bleb formation, ${ }^{26,29}$ whereas low WSS may trigger an inflammatory cell-mediated destructive remodeling pathway, akin to atherosclerotic (aortic aneurysm) development. The 2 hypothesized pathways can dominate different phases of IA development (including rupture) or even different parts of the same aneurysm sac at a given time.

Given such heterogeneity in aneurysmal hemodynamic-biologic mechanisms and manifestation, studies with a limited number of samples could be skewed to one side of the spectrum or the other. Overall, however, we think that after the aneurysm initiation phase (which has been determined to be driven by high WSS and high, positive WSS gradient ${ }^{14,15}$ ), aneurysm sac enlargement tends to continuously lower WSS, allowing inflammation to dominate the rest of IA development. Still, a smaller set of aneurysms may be continuously dominated by impinging flow because of their geometry; thus, their growth and rupture may be driven by high WSS through the noninflammatory pathway.

\section{Summary}

Collaborative efforts between computational researchers and clinicians have thrust image-based CFD to the forefront of emerging patient-specific IA evaluations. WSS-based hemodynamic metrics are promising predictors of IA growth and rupture. However, the rapid growth of this field has also generated inconsistent hemodynamic parameters and at times produced conflicting results, raising concerns of the clinical utility of image-based CFD.

The "high-versus-low WSS" controversy represents the pinnacle of this unsettling issue. Although it is not surprising to see inconsistent results from studies that are based on small datasets (typically 1-30 IA cases), ${ }^{8}$ it is disconcerting that larger studies with consistent WSS definitions have produced opposite results concerning the correlation of WSS with IA rupture. This suggests that perhaps the mechanistic heterogeneity underlying IA growth and rupture leads to different characteristics within IA datasets collected at different centers, thereby producing the inconsistent findings reported in the current literature.

We believe that the intrinsic complexity of aneurysm growth and rupture mechanisms could be a major cause of inconsistent findings. As elaborated on in Part $1,{ }^{11}$ high and low WSS can elicit different hemodynamic-biologic pathways of pathologic remodeling, driving IA growth and rupture and accounting for heterogeneous aneurysm phenotypes. As such, IA heterogeneity may need to be taken into account in future large-scale correlative hemodynamic IA studies. Computational fluid dynamics is not "confounding factor dissemination"; rather, it enables us to appreciate the complexity of IA pathophysiology driven by hemodynamics. To close the gap between CFD research and clinical utility, however, we must perform CFD more thoughtfully. We expect that both larger databases and better ways of categorizing and analyzing IAs, along with increased knowledge of the hemodynamic-mechanobiology of IA, will continue to define the role of hemodynamics in IA growth and rupture, leading to more accurate rupture risk assessments and improved clinical utility of CFD analyses.

\section{Future Directions}

Going forward, the heterogeneity of IA presentation and the proposed underlying hemodynamic mechanisms should be addressed in large, multi-center, CFD studies. Large databases could help to consolidate contradictory WSS findings, form conceptual models of IA pathophysiology, and build a foundation for more accurate clinical risk stratification models.

To facilitate the creation of such databases, CFD researchers should begin to standardize and unify aneurysm modeling procedures and hemodynamic variable definitions. ${ }^{8}$ Once these procedures are standardized, it will be possible to combine and compare datasets from different centers with assured consistency, which is required to conduct studies of aneurysm rupture risk that 
are valid across centers and populations. A common framework, available across the entire aneurysm research community, on which to study the connections between hemodynamics and aneurysm growth and rupture, will help accomplish this goal. On the other hand, we should also advance the clinical integration of user-friendly, streamlined computational tools at the point of care. Such clinically oriented IA analysis tools will enable clinicians to gain first-hand experience with CFD modeling, sharpen their intuition of treatment decisions and planning on the basis of flow visualization, and rapidly provide sorely needed constructive feedback and guidance to computational researchers. ${ }^{8}$

Mechanistic heterogeneity implies that different datasets, or subsets of datasets, could be dominated by different mechanisms of IA growth and rupture. Thus, whereas larger databases could increase the statistical prowess of predictive metrics, they also could produce more divergence. More sophisticated data mining and analytic methods will be required to categorize aneurysms. In accordance with the hypothesis that both high and low WSS can drive aneurysm growth and rupture through different biologic mechanisms, ${ }^{11}$ integrative hemodynamic parameters capturing both mechanisms could be explored as potentially better risk predictors. Alternatively, aneurysms could be categorized by location or type for the extrapolation of separate predictive markers. These foreseeable developments are expected to not only produce more accurate predictors of IA growth and rupture, but also consolidate the large number of predictive metrics suggested in the literature.

Modeling and analytic efforts must be directed by a better understanding of hemodynamic-biologic and mechanobiological mechanisms of IA growth and rupture. Ideally, both hemodynamic-biologic and mechanobiological mechanisms at play during IA natural history should be tested in experimental models, but endogenous models of IA growth and rupture do not currently exist. At the present time, efforts can be made to follow different types of IAs (eg, Type I and Type II, see Part $1^{11}$ ) in patients when possible, so that specific hemodynamic-biologic pathways related to aneurysm growth and rupture can be identified. Furthermore, study of the mechanobiology of the IA wall, for example, the interaction of aneurysm biology and wall mechanics, will help researchers better understand the mechanisms of IA growth and rupture that cannot be explained wholly by hemodynamics. ${ }^{9}$

It is important to recognize that most aneurysm datasets obtained clinically are composed of cross-sectional data. Bringing computational tools to the bedside can improve our ability to follow and analyze aneurysm hemodynamics and natural history longitudinally. Such data are vital not only for the validation of predictive models built from cross-sectional data but also for the development of new models that incorporate the progression of time. As proposed in Part $1,{ }^{11}$ hemodynamics may change roles throughout IA natural history. The advancement of IA wall mechanobiological measurements, combined with longitudinal aneurysm datasets, could enable a new class of computational tools, for example, fluid-solid-growth models, to simulate IA progression. ${ }^{45}$ Such longitudinal models could provide a general framework upon which researchers might explore aneurysm evolution and test hypotheses related to aneurysm growth and rupture, leading to more robust rupture risk assessments. ${ }^{9,45}$ Fur- thermore, by incorporating hemodynamics, mechanobiological wall mechanics, and geometry changes throughout IA natural history, fluid-solid-growth models could allow researchers to infer changing mechanisms during growth and rupture.

We hope that this review not only helps to delineate the current CFD literature to clarify the WSS controversy but also calls for worldwide interactions among CFD researchers and clinicians. Collaborative efforts will converge discrepancies in the field and ultimately lead to a greater understanding of the complex aneurysm pathophysiology, more robust risk predictors, and better patient care outcomes.

\section{ACKNOWLEDGMENTS}

We thank Dr Charles Strother for stimulating discussions.

Disclosures: Kenneth Snyder—RELATED: Grant: Toshiba, ${ }^{*}$ ev3*; Consulting Fee or Honorarium: Toshiba, ev3, Covidien; Support for Travel to Meetings for the Study or Other Purposes: Toshiba, ev3, Covidien; Other: Toshiba, ev3, Covidien; UNRELATED: Consultancy: Toshiba, ev3, Covidien; Grants/Grants Pending: Toshiba,* ev3*; Payment for Lectures (including service on speakers bureaus): Toshiba, ev3, Covidien; Other: Toshiba, ev3, Covidien. Hui Meng—RELATED: Grant: $\mathrm{NIH},{ }^{*}$ Toshiba, ${ }^{*}$ Comments: NIH Grant R01NS064592 for investigating pathogenesis of intracranial aneurysm; A grant from Toshiba Medical System Corp. for CFD study of aneurysm. ( ${ }^{*}$ money paid to institution).

\section{REFERENCES}

1. Jou LD, Wong G, Dispensa B, et al. Correlation between lumenal geometry changes and hemodynamics in fusiform intracranial aneurysms. AJNR Am J Neuroradiol 2005;26:2357-63

2. Acevedo-Bolton G, Jou LD, Dispensa BP, et al. Estimating the hemodynamic impact of interventional treatments of aneurysms: numerical simulation with experimental validation: technical case report. Neurosurgery 2006;59:E429-30

3. Sugiyama SI, Meng H, Funamoto K, et al. Hemodynamic analysis of growing intracranial aneurysms arising from a posterior inferior cerebellar artery. World Neurosurg 2012;78:462-68

4. Xiang J, Natarajan SK, Tremmel M, et al. Hemodynamic-morphologic discriminants for intracranial aneurysm rupture. Stroke 2011;42:144-52

5. Cebral JR, Mut F, Weir J, et al. Quantitative characterization of the hemodynamic environment in ruptured and unruptured brain aneurysms. AJNR Am J Neuroradiol 2011;32:145-51

6. Steinman DA. Computational modeling and flow diverters: a teaching moment. AJNR Am J Neuroradiol 2011;32:981-83

7. Kallmes DF. Point: CFD-computational fluid dynamics or confounding factor dissemination. AJNR Am J Neuroradiol 2012;33:395-96

8. Cebral JR, Meng H. Counterpoint: realizing the clinical utility of computational fluid dynamics-closing the gap. AJNR Am J Neuroradiol 2012;33:396-98

9. Robertson AM, Watton PN. Computational fluid dynamics in aneurysm research: critical reflections, future directions. AJNR Am J Neuroradiol 2012;33:992-95

10. Strother CM, Jiang J. Intracranial aneurysms, cancer, $\mathbf{x}$-rays, and computational fluid dynamics. AJNR Am J Neuroradiol 2012;33:991-92

11. Meng H, Tutino VM, Xiang J, et al. High WSS or low WSS? Complex interactions of hemodynamics with intracranial aneurysm initiation, growth, and rupture: toward a unifying hypothesis. AJNR AmJ Neuroradiol 2014;35:1254-62

12. Shojima M, Oshima M, Takagi $\mathrm{K}$, et al. Magnitude and role of wall shear stress on cerebral aneurysm: computational fluid dynamic study of 20 middle cerebral artery aneurysms. Stroke 2004;35:2500-05

13. Jou LD, Lee DH, Morsi $H$, et al. Wall shear stress on ruptured and 
unruptured intracranial aneurysms at the internal carotid artery. AJNR Am J Neuroradiol 2008;29:1761-67

14. Meng H, Wang Z, Hoi Y, et al. Complex hemodynamics at the apex of an arterial bifurcation induces vascular remodeling resembling cerebral aneurysm initiation. Stroke 2007;38:1924-31

15. Metaxa E, Tremmel M, Natarajan SK, et al. Characterization of critical hemodynamics contributing to aneurysmal remodeling at the basilar terminus in a rabbit model. Stroke 2010;41:1774-82

16. Malek AM, Alper SL, Izumo S. Hemodynamic shear stress and its role in atherosclerosis. JAMA 1999;282:2035-42

17. Boussel L, Rayz V, McCulloch C, et al. Aneurysm growth occurs at region of low wall shear stress: patient-specific correlation of hemodynamics and growth in a longitudinal study. Stroke 2008; 39:2997-3002

18. Tateshima S, Tanishita $\mathrm{K}$, Omura $\mathrm{H}$, et al. Intra-aneurysmal hemodynamics during the growth of an unruptured aneurysm: in vitro study using longitudinal CT angiogram database. AJNR Am J Neuroradiol 2007;28:622-27

19. Sforza DM, Putman CM, Tateshima S, et al. Hemodynamics characteristics of growing and stable aneurysms. ASME Summer Bioengineering Conference, Fajardo, Puerto Rico, June 20-23, 2012

20. Cebral JR, Castro MA, Burgess JE, et al. Characterization of cerebral aneurysms for assessing risk of rupture by using patient-specific computational hemodynamics models. AJNR Am J Neuroradiol 2005;26:2550-59

21. Hassan T, Timofeev EV, Saito T, et al. A proposed parent vessel geometry-based categorization of saccular intracranial aneurysms: computational flow dynamics analysis of the risk factors for lesion rupture. J Neurosurg 2005;103:662-80

22. Castro M, Putman C, Radaelli A, et al. Hemodynamics and rupture of terminal cerebral aneurysms. Acad Radiol 2009;16:1201-07

23. Castro MA, Putman CM, Sheridan MJ, et al. Hemodynamic patterns of anterior communicating artery aneurysms: a possible association with rupture. AJNR Am $J$ Neuroradiol 2009;30:297-302

24. Chien A, Castro MA, Tateshima S, et al. Quantitative hemodynamic analysis of brain aneurysms at different locations. AJNR Am J Neuroradiol 2009;30:1507-12

25. Chien A, Tateshima S, Sayre J, et al. Patient-specific hemodynamic analysis of small internal carotid artery-ophthalmic artery aneurysms. Surg Neurol 2009;72:444-50

26. Cebral JR, Sheridan M, Putman CM. Hemodynamics and bleb formation in intracranial aneurysms. AJNR Am J Neuroradiol 2010;31:304-10

27. Lu G, Huang L, Zhang XL, et al. Influence of hemodynamic factors on rupture of intracranial aneurysms: patient-specific 3D mirror aneurysms model computational fluid dynamics simulation. AJNR Am J Neuroradiol 2011;32:1255-61

28. Qian Y, Takao H, Umezu M, et al. Risk analysis of unruptured aneurysms using computational fluid dynamics technology: preliminary results. AJNR Am J Neuroradiol 2011;32:1948-55

29. Zhang $\mathrm{Y}, \mathrm{Mu} \mathrm{S}$, Chen J, et al. Hemodynamic analysis of intracranial aneurysms with daughter blebs. Eur Neurol 2011;66:359-67

30. Goubergrits L, Schaller J, Kertzscher U, et al. Statistical wall shear stress maps of ruptured and unruptured middle cerebral artery aneurysms. J R Soc Interface 2012;9:677-88

31. Takao H, Murayama $Y$, Otsuka $S$, et al. Hemodynamic differences between unruptured and ruptured intracranial aneurysms during observation. Stroke 2012;43:1436-39

32. Hoi $\mathrm{Y}$, Meng $\mathrm{H}$, Woodward SH, et al. Effects of arterial geometry on aneurysm growth: three-dimensional computational fluid dynamics study. J Neurosurg 2004;101:676-81

33. Shojima M, Oshima M, Takagi $K$, et al. Role of the bloodstream impacting force and the local pressure elevation in the rupture of cerebral aneurysms. Stroke 2005;36:1933-38

34. Valencia A, Morales H, Rivera R, et al. Blood flow dynamics in patient-specific cerebral aneurysm models: the relationship between wall shear stress and aneurysm area index. Med Eng Phys 2008;30:329-40

35. Omodaka S, Sugiyama $S$, Inoue $T$, et al. Local hemodynamics at the rupture point of cerebral aneurysms determined by computational fluid dynamics analysis. Cerebrovasc Dis 2012;34:121-29

36. Kawaguchi T, Kanamori M, Takazawa H, et al. Flow dynamics analysis in patients with a ruptured middle cerebral artery aneurysm: a case report. No Shinkei Geka 2011;39:281-86

37. Miura $Y$, Ishida F, Umeda $Y$, et al. Low wall shear stress is independently associated with the rupture status of middle cerebral artery aneurysms. Stroke 2013;44:519-21

38. Xu J, Yu Y, Wu X, et al. Morphological and hemodynamic analysis of mirror posterior communicating artery aneurysms. PloS One 2013;8:e55413

39. Antiga L, Piccinelli M, Botti L, et al. An image-based modeling framework for patient-specific computational hemodynamics. Med Biol Eng Comput 2008;46:1097-112

40. Geers AJ, Larrabide I, Radaelli AG, et al. Patient-specific computational hemodynamics of intracranial aneurysms from 3D rotational angiography and CT angiography: an in vivo reproducibility study. AJNR Am J Neuroradiol 2011;32:581-86

41. Morales HG, Kim M, Vivas EE, et al. How do coil configuration and packing density influence intra-aneurysmal hemodynamics? AJNR Am J Neuroradiol 2011;32:1935-41

42. Marzo A, Singh P, Larrabide I, et al. Computational hemodynamics in cerebral aneurysms: the effects of modeled versus measured boundary conditions. Ann Biomed Eng 2011;39:884-96

43. Cebral JR, Mut F, Weir J, et al. Association of hemodynamic characteristics and cerebral aneurysm rupture. AJNR Am J Neuroradiol 2011;32:264-70

44. Steinman DA, Milner JS, Norley CJ, et al. Image-based computational simulation of flow dynamics in a giant intracranial aneurysm. AJNR Am J Neuroradiol 2003;24:559-66

45. Humphrey JD, Taylor CA. Intracranial and abdominal aortic aneurysms: similarities, differences, and need for a new class of computational models. Annu Rev Biomed Eng 2008;10:221-46

46. Nixon AM, Gunel M, Sumpio BE. The critical role of hemodynamics in the development of cerebral vascular disease. J Neurosurg 2010;112:1240-53

47. Ota R, Kurihara C, Tsou TL, et al. Roles of matrix metalloproteinases in flow-induced outward vascular remodeling. J Cereb Blood Flow Metab 2009;29:1547-58

48. Frosen J, Tulamo R, Paetau A, et al. Saccular intracranial aneurysm: pathology and mechanisms. Acta Neuropathol 2012;123:773-86

49. Wang Z, Kolega J, Hoi Y, et al. Molecular alterations associated with aneurysmal remodeling are localized in the high hemodynamic stress region of a created carotid bifurcation. Neurosurgery 2009;65:169-77

50. Kolega J, Gao L, Mandelbaum M, et al. Cellular and molecular responses of the basilar terminus to hemodynamics during intracranial aneurysm initiation in a rabbit model. $J$ Vasc Res 2011;48:429-42

51. Watton PN, Selimovic A, Raberger NB, et al. Modelling evolution and the evolving mechanical environment of saccular cerebral aneurysms. Biomech Modeling Mechanobiol 2011;10:109-32

52. Cardenes R, Pozo JM, Bogunovic H, et al. Automatic aneurysm neck detection using surface Voronoi diagrams. IEEE Trans Med Imaging 2011;30:1863-76

53. Larrabide I, Cruz Villa-Uriol M, Cardenes R, et al. Three-dimensional morphological analysis of intracranial aneurysms: a fully automated method for aneurysm sac isolation and quantification. Med Physics 2011;38:2439-49

54. Dhar S, Tremmel M, Mocco J, et al. Morphology parameters for intracranial aneurysm rupture risk assessment. Neurosurgery 2008;63:185-96

55. Rahman M, Smietana J, Hauck E, et al. Size ratio correlates with intracranial aneurysm rupture status: a prospective study. Stroke 2010;41:916-20 
56. Ma D, Tremmel M, Paluch RA, et al. Size ratio for clinical assessment of intracranial aneurysm rupture risk. Neurol Res 2010; 32:482-86

57. Dolan JM, Meng H, Singh S, et al. High fluid shear stress and spatial shear stress gradients affect endothelial proliferation, survival, and alignment. Ann Biomed Eng 2011;39:1620-31

58. LaMack JA, Friedman MH. Individual and combined effects of shear stress magnitude and spatial gradient on endothelial cell gene expression. Am J Physiol Heart Circ Physiol 2007;293: H2853-59

59. He X, Ku DN. Pulsatile flow in the human left coronary artery bifurcation: average conditions. J Biomech Eng 1996;118:74-82
60. Shimogonya $\mathrm{Y}$, Ishikawa $\mathrm{T}$, Imai $\mathrm{Y}$, et al. Can temporal fluctuation in spatial wall shear stress gradient initiate a cerebral aneurysm? A proposed novel hemodynamic index, the gradient oscillatory number (GON). J Biomech 2009;42:550-54

61. Chien A, Tateshima S, Castro M, et al. Patient-specific flow analysis of brain aneurysms at a single location: comparison of hemodynamic characteristics in small aneurysms. Med Biol Eng Comput 2008;46:1113-20

62. Kawaguchi T, Nishimura S, Kanamori M, et al. Distinctive flow pattern of wall shear stress and oscillatory shear index: similarity and dissimilarity in ruptured and unruptured cerebral aneurysm blebs. J Neurosurg 2012;117:774-80 